\section{A new administration}

\author{
Keith Barton, ${ }^{1}$ James Chodosh, ${ }^{2}$ Jost Jonas ${ }^{3}$
}

As incoming Editors-in-Chief of the British Journal of Ophthalmology we thank Profs Harminder Dua and Arun Singh for their careful stewardship of the journal. Following in their footsteps is a formidable challenge but thanks to their commitment, the unstinting support of their editorial board and legions of anonymous reviewers we have inherited a great gift.

The BJO, though a national treasure with a long tradition, is truly a global publication. The quality of the BJO reflects the very high quality of ophthalmology care and training, not only in the UK, but also around the world. We aim to maintain this high quality in the face of competition from the recent deluge of online journals and ensure that research-active clinicians and scientists continue to aspire to publish in the BJO.

Our goals as Editors-in-Chief are to maintain high standards in published research, to further improve the relevance

\footnotetext{
${ }^{1} \mathrm{NIHR}$ Biomedical Research Centre for Ophthalmology, Moorfields Eye Hospital and UCL Institute of Ophthalmology, London, UK; ${ }^{2}$ Department of Ophthalmology, Boston, Massachusetts, US; ${ }^{3}$ Department of Ophthalmology, Mannheim, Germany Correspondence to Keith Barton, Moorfields Eye Hospital, ECIV 2PD London, UK;

BJO@keithbarton.co.uk
}

and attractiveness of the journal to both its contributors and its readers. Specific measures to achieve these goals include improving the turnaround time of reviews, speeding up the time to online publication, and improving communication between the Editors-in-Chief and BJO contributors.

Manuscripts deemed unlikely to achieve acceptance will be returned to authors quickly to facilitate resubmission elsewhere. Our editorial policy will continue to oppose commercial and professional bias. We will solicit the highest quality review articles from leaders in the field in order to provide expert comprehensive coverage of topical areas. We will aim to attract more diverse content including studies performed across Africa and Asia; we particularly encourage authors from these regions to submit manuscripts.

We three cover different specialties within the field of ophthalmology and from different countries. Keith Barton is a consultant ophthalmic surgeon in the glaucoma service at Moorfields Eye Hospital in London, honorary reader in the Institute of Ophthalmology, University College London, and Hong Leong Visiting Professor at the National University of Singapore. He specializes in the surgical management of glaucoma and especially secondary glaucomas. James
Chodosh is the David Glendenning Cogan Professor of Ophthalmology at Harvard Medical School, and Associate Director of the Cornea and Refractive Surgery Service at the Massachusetts Eye and Ear Infirmary, in Boston, Massachusetts. His clinical and research interests are in infectious eye diseases and the surgical restoration of complex cases of corneal blindness. Jost Jonas is Professor at the Department of Ophthalmology of the University of Heidelberg, Germany and at the Beijing Institute of Ophthalmology, China; he is a comprehensive ophthalmologist.

Overall, we will strive to act as academics as defined by Plato; for whom a true academic was one who in both theory and practice, in both thinking and acting, holds up the primacy of intellect over monetary issues and who subordinates power to justice. We hope to produce interesting and informative articles for an interested and informed readership. We look forward to receiving your submissions.

Competing interests None.

Provenance and peer review Commissioned; internally peer reviewed.

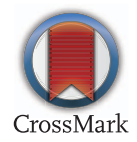

To cite Barton K, Chodosh J, Jonas J. Br J Ophthalmol 2014;98:715.

Br J Ophthalmol 2014;98:715.

doi:10.1136/bjophthalmol-2014-305452 